\title{
Horadam $2^{k}$-ions
}

\author{
Melih Göcen and Yüksel Soykan
}

\begin{abstract}
In this paper, we generalize Fibonacci quaternion, octonion, sedenion, trigintaduonion, etc. and define Horadam $2^{k}$-ions and investigate their properties. Each Horadam (such as Fibonacci, Lucas, Pell) quaternions, octonions and sedenions are Horadam $2^{k}$-ions. We also present connection to some earlier works.
\end{abstract}

Key Words: quaternions, octonions, sedenions, Horadam numbers, Fibonacci quaternion, Cayley-Dickson algebra.

2010 Mathematics Subject Classification: 11B39, 11B83.

\section{Introduction}

In this work we generalize Fibonacci quaternion, octonion, sedenion, trigintaduonion and so on. In section 1 we give definition of $2^{k}$-ions and in section 2 we give definition of Horadam numbers which is generalization of Fibonacci numbers and contains Fibonacci, Lucas, Pell, Jacobsthal and all other second order sequences. In section 3, we introduce Horadam $2^{k}$-ions which contains Fibonacci, Lucas, Pell, Jacobsthal quaternions (octonions and sedenions). Moreover, in that section we give Binet formula, generating function, norm value of Horadam $2^{k}$-ions and also Catalan identity, Cassini identity, d'Ocagne identity and a summation formula. In section 4 we present matrix methods in Horadam $2^{k}$-ions.

In the following, we will briefly present important and very known algebra: Cayley-Dickson algebra.

In 1843, William Rowan Hamilton discovered the quaternions $(\mathbb{H})$, which is a $4=2^{2}$-dimensional algebra over $\mathbb{R}$. This algebra is an associative and a noncommutative algebra. In 1843, John Graves discovered the octonions $(\mathbb{O})$, a $8=2^{3}$-dimensional algebra over $\mathbb{R}$ which is a nonassociative and a noncommutative algebra. In 1845, these algebras were rediscovered by Arthur Cayley. They are also known as the Cayley numbers. This process, of passing from $\mathbb{R}$ to $\mathbb{C}$, from $\mathbb{C}$ to $\mathbb{H}$ and from $\mathbb{H}$ to $\mathbb{O}$ was generalized to algebras over arbitrary fields and rings. It is called the Cayley-Dickson doubling process or the Cayley-Dickson process. The next doubling process applied to $\mathbb{O}$ then produces an algebra $\mathbb{S}\left(\operatorname{dim} 2^{4}=16\right)$ called the sedenions. This doubling process can be extended beyond the sedenions to form what are known as the $2^{k}$-ions (see for example [1], [18], [22]). 
Now, we explain this doubling process. The Cayley-Dickson algebras are a sequence $A_{0}, A_{1}, \ldots$ of nonassociative $\mathbb{R}$-algebras with involution. The term "conjugation" can be used to refer to the involution because it generalizes the usual conjugation on the complex numbers. For a full explanation of the basic properties of Cayley-Dickson algebras, see [1]. Cayley-Dickson algebras are defined inductively. We begin by defining $A_{0}$ to be $\mathbb{R}$. Given $A_{k-1}$, the algebra $A_{k}$ is defined additively to be $A_{k-1} \times A_{k-1}$. Conjugation in $A_{k}$ is defined by

$$
\overline{(a, b)}=(\bar{a},-b)
$$

and multiplication is defined by

$$
(a, b)(c, d)=(a c-\bar{d} b, d a+b \bar{c})
$$

and addition is defined by componentwise as

$$
(a, b)+(c, d)=(a+c, b+d) .
$$

$A_{k}$ has dimension $N=2^{k}$ as an $\mathbb{R}$-vector space. If we set, as usual, $\|x\|=\sqrt{\operatorname{Re}(x \bar{x})}$ for $x \in A_{k}$ then $x \bar{x}=\bar{x} x=\|x\|^{2}$.

For specific $k$, how $2^{k}$-ions are called, is given in the following table.

Table 1.

\begin{tabular}{|l|l|l|l|l|l|}
\hline$k$ & 2 & 3 & 4 & 5 & $\ldots$ \\
\hline $2^{k}$-ions & Quaternions & Octonions & Sedenions & Trigintaduonions & $\ldots$ \\
\hline
\end{tabular}

Now, for a fixed $k$ suppose that $B_{N}=\left\{e_{i} \in A_{k}: i=0,1,2, \ldots, N-1\right\}$ is the basis for $A_{k}$, where $N=2^{k}$ is the dimension of $A_{k}, e_{0}$ is the identity (or unit) and $e_{1}, e_{2}, \ldots, e_{N-1}$ are called imaginaries. Then a $2^{k}$-ions $S \in A_{k}$ can be written as

$$
S=\sum_{i=0}^{N-1} a_{i} e_{i}=a_{0}+\sum_{i=1}^{N-1} a_{i} e_{i}
$$

where $a_{0}, a_{1}, \ldots, a_{N-1}$ are all real numbers. Here $a_{0}$ is called the real part of $S$ and $\sum_{i=1}^{N-1} a_{i} e_{i}$ is called its imaginary part.

Addition of $2^{k}$-ions is defined as componentwise and multiplication is defined as follows: if $S_{1}, S_{2} \in A_{k}$ then we have

$$
S_{1} S_{2}=\left(\sum_{i=0}^{N-1} a_{i} e_{i}\right)\left(\sum_{i=0}^{N-1} b_{i} e_{i}\right)=\sum_{i, j=0}^{N-1} a_{i} b_{j}\left(e_{i} e_{j}\right) .
$$

The operations requiring for the multiplication in (1.2) are quite a lot. At the hypercomplex algebra the most time-consuming operation is the multiplication of two hypercomplex numbers. This is because the multiplication of two $N$-dimensional hypercomplex numbers requires $N^{2}$ real multiplications and $N(N-1)$ real additions. For example, the computation of a trigintaduonions multiplication (product) using the naive method requires 1024 real multiplications and 992 real additions, while an algorithm which is given in [5] 
can compute the same result in only 498 real multiplications (or multipliers - in hardware implementation case) and 943 real additions, for details see [5].

Using direct multiplication, the number of the operations requiring for the multiplication of two (specific) $2^{k}$-ions is given in the following table.

Table 2.

\begin{tabular}{cccc}
\hline $2^{k}$-ions & Computation method & Multiplications & Additions \\
\hline Quaternions & Based on expression (1.2) & 16 & 12 \\
Octonions & Based on expression (1.2) & 64 & 56 \\
Sedenions & Based on expression (1.2) & 256 & 240 \\
Trigintaduonions & Based on expression (1.2) & 1024 & 992
\end{tabular}

Efficient algorithms for the multiplication of quaternions, octonions, sedenions, and trigintaduonions with reduced number of real multiplications is already exist and results of synthesizing an efficient algorithm of computing the two $2^{k}$-ions product are presented in the following table.

Table 3.

\begin{tabular}{cccc}
\hline $2^{k}$-ions & Computation method & Multiplications & Additions \\
\hline Quaternions & Algorithm in [24] & 8 & - \\
Octonions & Algorithm in [3] & 32 & 88 \\
Sedenions & Algorithm in [4] & 122 & 298 \\
Trigintaduonions & Algorithm in [5] & 498 & 943 \\
\hline
\end{tabular}

\section{Horadam Numbers}

Now let us recall the definition of Horadam numbers. In 1965, Horadam [15] defined a generalization of Fibonacci sequence, that is, he defined a second-order linear recurrence sequence $\left\{W_{n}(a, b ; p, q)\right\}$, or simply $\left\{W_{n}\right\}$, as follows:

$$
W_{n}=p W_{n-1}+q W_{n-2} ; \quad W_{0}=a, W_{1}=b, \quad(n \geq 2)
$$

where $a, b, p$ and $q$ are arbitrary real numbers, see also Horadam [13], [16] and [17], . Now these numbers $\left\{W_{n}(a, b ; p, q)\right\}$ are called Horadam numbers.

For some specific values of $a, b, p$ and $q$, it is worth presenting these special Horadam numbers in a table as a specific name.

Table 4. 


\begin{tabular}{ccc}
\hline Name of sequence & $W_{n}(a, b ; p, q)$ & Binet Formula \\
\hline Fibonacci & $W_{n}(0,1 ; 1,1)=F_{n}$ & $\frac{\left(\frac{1+\sqrt{5}}{2}\right)^{n}-\left(\frac{1-\sqrt{5}}{2}\right)^{n}}{{ }^{\sqrt{5}}}$ \\
Lucas & $W_{n}(2,1 ; 1,1)=L_{n}$ & $\left(\frac{1+\sqrt{5}}{2}\right)^{n}+\left(\frac{1-\sqrt{5}}{2}\right)^{n}$ \\
Pell & $W_{n}(0,1 ; 2,1)=P_{n}$ & $\frac{(1+\sqrt{2})^{n}-(1-\sqrt{2})^{n}}{2 \sqrt{2}}$ \\
Pell-Lucas & $W_{n}(2,2 ; 2,1)=p_{n}$ & $(1+\sqrt{2})^{n}+(1-\sqrt{2})^{n}$ \\
Jacobsthal & $W_{n}(0,1 ; 1,2)=J_{n}$ & $\frac{2^{n}-(-1)^{n}}{3}$ \\
Jacobsthal-Lucas & $W_{n}(2,1 ; 1,2)=j_{n}$ & $2^{n}+(-1)^{n}$ \\
\hline
\end{tabular}

We can list some important properties of Horadam numbers that are needed.

- Binet formula of Horadam sequence can be calculated using its characteristic equation which is given as

$$
t^{2}-p t-q=0
$$

The roots of characteristic equation are

$$
\alpha=\frac{p+\sqrt{\Delta}}{2}, \beta=\frac{p-\sqrt{\Delta}}{2} .
$$

where $\Delta=p^{2}+4 q$. Using these roots and the recurrence relation Binet formula can be given as follows

$$
W_{n}=\frac{A \alpha^{n}-B \beta^{n}}{\alpha-\beta}
$$

where $A=b-a \beta$ and $B=b-a \alpha$.

- The generating function for Horadam numbers is

$$
g(t)=\frac{W_{0}+\left(W_{1}-p W_{0}\right) t}{1-p t-q t^{2}}
$$

- The Cassini identity for Horadam numbers is

$$
W_{n+1} W_{n-1}-W_{n}^{2}=q^{n-1}\left(p W_{0} W_{1}-W_{1}^{2}-W_{0}^{2} q\right) .
$$

- A summation formula for Horadam numbers is

$$
\sum_{i=0}^{n} W_{i}=\frac{W_{1}-W_{0}(p-1)+q W_{n}-W_{n+1}}{1-p-q} .
$$

- For $\Delta=p^{2}+4 q>0, \alpha$ and $\beta$ are reals and $\alpha \neq \beta$. Note also that

$$
\alpha^{2}=\alpha \sqrt{\Delta}-q
$$

and

$$
\beta^{2}=-\beta \sqrt{\Delta}-q .
$$




$$
\begin{aligned}
& A \alpha^{n}=\alpha W_{n}+q W_{n-1}, \\
& B \beta^{n}=\beta W_{n}+q W_{n-1} .
\end{aligned}
$$

\section{Horadam $2^{k}$-ions}

In this section we introduce Horadam $2^{k}$-ions and present their important properties. We give Binet formula, generating function, Cassini identity, summation formula and norm of these $2^{k}$-ions. First, we give some information about (Horadam) quaternion, octonion, sedenion and trigintaduonion sequences from the literature.

- Horadam [14] introduced $n$th Fibonacci and $n$th Lucas quaternions as

$$
Q_{n}=F_{n}+F_{n+1} e_{1}+F_{n+2} e_{2}+F_{n+3} e_{3}=\sum_{s=0}^{3} F_{n+s} e_{s}
$$

and

$$
R_{n}=L_{n}+L_{n+1} e_{1}+L_{n+2} e_{2}+L_{n+3} e_{3}=\sum_{s=0}^{3} L_{n+s} e_{s}
$$

respectively, where $F_{n}$ and $L_{n}$ are the $n$th Fibonacci and Lucas numbers respectively. See also Halici and Karataş [12], and Polatlı [23].

- Various families of octonion number sequences (such as Fibonacci octonion, Pell octonion, Jacobsthal octonion, and third order Jacobsthal octonion) have been defined and studied by a number of authors in many different ways. For example, Keçilioglu and Akkuş [21] introduced the Fibonacci and Lucas octonions as

$$
Q_{n}=\sum_{s=0}^{7} F_{n+s} e_{s}
$$

and

$$
R_{n}=\sum_{s=0}^{7} L_{n+s} e_{s}
$$

respectively, where $F_{n}$ and $L_{n}$ are the $n$th Fibonacci and Lucas numbers respectively. In [10], Çimen and İpek introduced Jacobsthal octonions and Jacobsthal-Lucas octonions. See also Tasci [26] for k-Jacobsthal and k-Jacobsthal-Lucas Quaternions and see İpek and Çimen [19] and Karataş and Halici [20] for Horadam type octonions. Moreover, we refer to Szynal-Liana and Wlock [25] for Pell quaternions and Pell octonions and Catarino [6] for Modified Pell and Modified k-Pell Quaternions and Octonions.

- A number of authors have been defined and studied sedenion number sequences (such as second order sedenions: Fibonacci sedenion, k-Pell and k-Pell-Lucas sedenions, Jacobsthal and JacobsthalLucas sedenions). For example, Bilgici, Tokeşer and Ünal [2] introduced the Fibonacci and Lucas 
sedenions as

$$
\widehat{F}_{n}=\sum_{s=0}^{15} F_{n+s} e_{s}
$$

and

$$
\widehat{L}_{n}=\sum_{s=0}^{15} L_{n+s} e_{s}
$$

respectively, where $F_{n}$ and $L_{n}$ are the $n$th Fibonacci and Lucas numbers respectively. In [7], Catarino introduced k-Pell and k-Pell-Lucas sedenions. In [9], Çimen and İpek introduced Jacobsthal and Jacobsthal-Lucas sedenions.

- Gül [11] introduced the k-Fibonacci and k-Lucas trigintaduonions as

$$
T F_{k, n}=\sum_{s=0}^{31} F_{k, n+s} e_{s}
$$

and

$$
T L_{k, n}=\sum_{s=0}^{31} L_{k, n+s} e_{s}
$$

respectively, where $F_{k, n}$ and $L_{k, n}$ are the $n$th k-Fibonacci and k-Lucas numbers respectively.

Definition 1. The Horadam $2^{k}$-ions sequence $\left\{\widehat{W}_{n}\right\}_{n \geq 0}$ is defined by the following recurrence relation:

$$
\widehat{W}_{n}=\sum_{s=0}^{N-1} W_{n+s} e_{s}
$$

where $W_{n}$ is the nth generalized Horadam number.

Note that from the definition of (2.1) and (3.1) we have the following recurrence relation:

$$
\widehat{W}_{n}=p \widehat{W}_{n-1}+q \widehat{W}_{n-2}
$$

Firstly, we present the Binet formula. This formula is very useful for finding desired Horadam $2^{k}$-ions and takes part at many theorems' proof. From now on, for fixed $k$ (so that the dimension of Horadam $2^{k}$-ions is $2^{k}=N$ ) we fixed the following notations:

$$
\widehat{\alpha}=\sum_{s=0}^{N-1} \alpha^{s} e_{s}, \quad \widehat{\beta}=\sum_{s=0}^{N-1} \beta^{s} e_{s} .
$$

Theorem 1. The Binet formula for Horadam $2^{k}$-ions is

$$
\widehat{W}_{n}=\frac{A \widehat{\alpha} \alpha^{n}-B \widehat{\beta} \beta^{n}}{\alpha-\beta} .
$$


Proof. Repeated use of (2.2) in (3.1) enable us to write:

$$
\begin{aligned}
\widehat{W}_{n}= & \sum_{s=0}^{N-1} W_{n+s} e_{s}=\left(\frac{A \alpha^{n}-B \beta^{n}}{\alpha-\beta}\right) e_{0}+\left(\frac{A \alpha^{n+1}-B \beta^{n+1}}{\alpha-\beta}\right) e_{1} \\
& +\left(\frac{A \alpha^{n+2}-B \beta^{n+2}}{\alpha-\beta}\right) e_{2}+\ldots+\left(\frac{A \alpha^{n+N-1}-B \beta^{n+N-1}}{\alpha-\beta}\right) e_{N-1} \\
= & \frac{A \widehat{\alpha} \alpha^{n}-B \widehat{\beta} \beta^{n}}{\alpha-\beta} .
\end{aligned}
$$

Alternative Proof of Theorem 1: We use the following identities:

$$
\begin{aligned}
& A \alpha^{n}=\alpha W_{n}+q W_{n-1}, \\
& B \beta^{n}=\beta W_{n}+q W_{n-1} .
\end{aligned}
$$

Note that

$$
\begin{aligned}
\alpha \widehat{W}_{n}+q \widehat{W}_{n-1} & =\alpha\left(W_{n}+W_{n+1} e_{1}+\ldots+W_{n+N-1} e_{N-1}\right)+q\left(W_{n-1}+W_{n} e_{1}+\ldots+W_{n+N-2} e_{N-1}\right) \\
& =\left(\alpha W_{n}+q W_{n-1}\right) e_{0}+\left(\alpha W_{n+1}+q W_{n}\right) e_{1}+\ldots+\left(\alpha W_{n+N-1}+q W_{n+N-2}\right) e_{N-1}
\end{aligned}
$$

From the identity $A \alpha^{n}=\alpha W_{n}+q W_{n-1}$ for $n$th Horadam number $W_{n}$, we have

$$
\alpha \widehat{W}_{n}+q \widehat{W}_{n-1}=A \widehat{\alpha} \alpha^{n}
$$

Similarly, we obtain

$$
\beta \widehat{W}_{n}+q \widehat{W}_{n-1}=B \widehat{\beta} \beta^{n}
$$

Substracting (3.5) from (3.4), we have

$$
\alpha \widehat{W}_{n}-\beta \widehat{W}_{n}=A \widehat{\alpha} \alpha^{n}-B \widehat{\beta} \beta^{n}
$$

So this proves (3.3).

For some particular cases of Binet formulas for Horadam $2^{k}$-ions, see Tables 5,6,7 and 8.

It is well known that for Horadam $2^{k}$-ions $\widehat{W}_{n}$ defined by (3.1) the ordinary generating function is $g(t)=\sum_{n=0}^{\infty} \widehat{W}_{n} t^{n}$. In the following theorem we present the generating function for Horadam $2^{k}$-ions.

THEOREM 2. The generating function for Horadam $2^{k}$-ions is

$$
g(t)=\frac{\widehat{W}_{0}+\left(\widehat{W}_{1}-p \widehat{W}_{0}\right) t}{1-p t-q t^{2}}
$$

Proof. Let

$$
g(t)=\sum_{n=0}^{\infty} \widehat{W}_{n} t^{n}
$$


be generating function of Horadam $2^{k}$-ions. Then using definition of Horadam $2^{k}$-ions, and substracting $p t g(t)$ and $q t^{2} g(t)$ from $g(t)$ we obtain (note the shift in the index $n$ in the third line)

$$
\begin{aligned}
\left(1-p t-q t^{2}\right) g(t) & =\sum_{n=0}^{\infty} \widehat{W}_{n} t^{n}-p t \sum_{n=0}^{\infty} \widehat{W}_{n} t^{n}-q t^{2} \sum_{n=0}^{\infty} \widehat{W}_{n} t^{n} \\
& =\sum_{n=0}^{\infty} \widehat{W}_{n} t^{n}-p \sum_{n=0}^{\infty} \widehat{W}_{n} t^{n+1}-q \sum_{n=0}^{\infty} \widehat{W}_{n} t^{n+2} \\
& =\sum_{n=0}^{\infty} \widehat{W}_{n} t^{n}-p \sum_{n=1}^{\infty} \widehat{W}_{n-1} t^{n}-q \sum_{n=2}^{\infty} \widehat{W}_{n-2} t^{n} \\
& =\left(\widehat{W}_{0}+\widehat{W}_{1} t\right)-p \widehat{W}_{0} t+\sum_{n=2}^{\infty}\left(\widehat{W}_{n}-p \widehat{W}_{n-1}-q \widehat{W}_{n-2}\right) t^{n} \\
& =\widehat{W}_{0}+\left(\widehat{W}_{1}-p \widehat{W}_{0}\right) t .
\end{aligned}
$$

Rearranging above equation, we get

$$
g(t)=\frac{\widehat{W}_{0}+\left(\widehat{W}_{1}-p \widehat{W}_{0}\right) t}{1-p t-q t^{2}} .
$$

For Horadam $2^{k}$-ions $\widehat{W}_{n}$ defined by (3.1), the exponential generating function is defined by $e(t)=$ $\sum_{n=0}^{\infty} \widehat{W}_{n} \frac{t^{n}}{n !}$

Theorem 3. For $\widehat{W}_{n}$, we have

$$
e(t)=\frac{A \widehat{\alpha} e^{\alpha t}-B \widehat{\beta} e^{\beta t}}{\alpha-\beta} .
$$

Proof. Using (3.3) in $e(t)=\sum_{n=0}^{\infty} \widehat{W}_{n} \frac{t^{n}}{n !}$, we obtain

$$
e(t)=\sum_{n=0}^{\infty}\left(\frac{A \widehat{\alpha} \alpha^{n}-B \widehat{\beta} \beta^{n}}{\alpha-\beta}\right) \frac{t^{n}}{n !} .
$$

Combining the formula $e^{t}=\sum_{n=0}^{\infty} \frac{t^{n}}{n !}$ and (3.9), we get (3.8).

There are three well-known identities for Fibonacci type numbers, namely, Catalan's, Cassini's, and d'Ocagne's identities. The proofs of these identities are based on Binet formulas. We can obtain these types of identities for Horadam $2^{k}$-ions using the Binet formulas derived above. Two different Catalan's identities for Horadam $2^{k}$-ions are given in the next theorem.

Theorem 4 (Catalan's Identity). For any integers $m$ and $n$ we have

(a):

$$
\widehat{W}_{m-n} \widehat{W}_{m+n}-\widehat{W}_{m}^{2}=\frac{A B(\alpha \beta)^{m}\left(\beta^{-n}-\alpha^{-n}\right)\left(\beta^{n} \widehat{\alpha} \widehat{\beta}-\alpha^{n} \widehat{\beta} \widehat{\alpha}\right)}{(\alpha-\beta)^{2}}
$$

(b):

$$
\widehat{W}_{m+n} \widehat{W}_{m-n}-\widehat{W}_{m}^{2}=\frac{A B(\alpha \beta)^{m}\left(\beta^{-n}-\alpha^{-n}\right)\left(\beta^{n} \widehat{\beta} \widehat{\alpha}-\alpha^{n} \widehat{\alpha} \widehat{\beta}\right)}{(\alpha-\beta)^{2}}
$$


Proof. By using Binet formula, we obtain

$$
\widehat{W}_{m-n} \widehat{W}_{m+n}-\widehat{W}_{m}^{2}=\frac{A \widehat{\alpha} \alpha^{m-n}-B \widehat{\beta} \beta^{m-n}}{\alpha-\beta} \frac{A \widehat{\alpha} \alpha^{m+n}-B \widehat{\beta} \beta^{m+n}}{\alpha-\beta}-\left(\frac{A \widehat{\alpha} \alpha^{m}-B \widehat{\beta} \beta^{m}}{\alpha-\beta}\right)^{2} .
$$

After necessary calculations we get the identity (3.10). Similarly we obtain (3.11).

Taking $n=1$ in Catalan's identitity we obtain Cassini's identitity for Horadam $2^{k}$-ions. In the next theorem, we state two different Cassini identities.

Corollary 1 (Cassini's Identitity). For any integer $m$, we have

(a):

$$
\widehat{W}_{m-1} \widehat{W}_{m+1}-\widehat{W}_{m}^{2}=\frac{A B(\alpha \beta)^{m-1}(\beta \widehat{\alpha} \widehat{\beta}-\alpha \widehat{\beta} \widehat{\alpha})}{\alpha-\beta}
$$

(b):

$$
\widehat{W}_{m+1} \widehat{W}_{m-1}-\widehat{W}_{m}^{2}=\frac{A B(\alpha \beta)^{m-1}(\beta \widehat{\beta} \widehat{\alpha}-\alpha \widehat{\alpha} \widehat{\beta})}{\alpha-\beta}
$$

Note that from non-commutativity $\widehat{\alpha} \widehat{\beta} \neq \widehat{\beta} \widehat{\alpha}$ and so $\beta \widehat{\alpha} \widehat{\beta}-\alpha \widehat{\beta} \widehat{\alpha} \neq \widehat{\alpha} \widehat{\beta}(\beta-\alpha)$.

We emphasize some particular cases of Binet formulas, generating functions and Cassini's identities for Horadam $2^{k}$-ions:

- Let $a=0, b=p=q=1$. For $k=2, k=3, k=4$ and $k=5$ we get the Binet formula, generating function, Cassini's identitity for Fibonacci quaternions which is given by Halici and Karataş in [12], the Binet formula, generating function, Cassini's identitity for Fibonacci octonions which is given by Keçilioğlu and Akkuş in [21] or Halici and Karataş [12], the Binet formula, generating function, Cassini's identitity for Fibonacci sedenions which is given by Bilgici, Tokeşer and Ünal [2], and the Binet formula, generating function, Cassini's identitity for Fibonacci trigintaduonions which is given by Gül in [11], respectively.

- Let $a=2, b=p=q=1$. For $k=2, k=3, k=4$ and $k=5$ we obtain the Binet formula, generating function, Cassini's identitity for Lucas quaternions which is given by Halici and Karataş in [12], the Binet formula, generating function, Cassini's identitity for Lucas octonions which is given by Halici and Karataş in [12], the Binet formula, generating function, Cassini's identitity for Lucas sedenions which is given by Bilgici, Tokeşer and Ünal in [2], and the Binet formula, generating function, Cassini's identitity for Lucas trigintaduonions which is given by Gül in [11], respectively.

- Let $a=0, b=1, p=2, q=1$. For $k=2, k=3$, and $k=4$, we get the Binet formula, generating function, Cassini's identitity for Pell quaternions which is given by Szynal-Liana and Wloch in [25], the Binet formula, generating function, Cassini's identitity for Pell octonions which is given by Szynal-Liana and Wloch in [25] and the Binet formula, generating function, Cassini's identitity for Pell sedenions which is given by Catarino in [7], respectively. 
- Let $a=0, b=p=1, q=2$. For $k=2, k=3$, and $k=4$, we obtain the Binet formula, generating function, Cassini's identitity for Jacobsthal quaternions which is given by Tasci in [26], the Binet formula, generating function, Cassini's identitity for Jacobsthal octonions which is given by Cimen and İpek in [10] and the Binet formula, generating function, Cassini's identitity for Jacobsthal sedenions which is given by Çimen and İpek in [9], respectively.

We give the next four tables to show the differences of the formulas for particular case even they looks the same.

In the following table we present Cassini's identities and Binet formulas for some Horadam (Fibonacci, Lucas, Pell, Jacobsthal) quaternions:

Table 5.

\begin{tabular}{ccc}
\hline Horadam 2 2 -ions: & $\widehat{W}_{m-1} \widehat{W}_{m+1}-\widehat{W}_{m}^{2}$ & Binet Formula \\
\hline Fibonacci Quaternion & $\frac{(-1)^{m-1}\left(\left(\frac{1-\sqrt{5}}{2}\right) \widehat{\alpha} \widehat{\beta}-\left(\frac{1+\sqrt{5}}{2}\right) \widehat{\beta} \widehat{\alpha}\right)}{\sqrt{5}}$ & $\frac{\widehat{\alpha}\left(\frac{1+\sqrt{5}}{2}\right)^{n}-\widehat{\beta}\left(\frac{1-\sqrt{5}}{2}\right)^{n}}{\sqrt{5}}$ \\
Lucas Quaternion & $\frac{-5(-1)^{m-1}\left(\left(\frac{1-\sqrt{5}}{2}\right) \widehat{\alpha}-\left(\frac{1+\sqrt{5}}{2}\right) \widehat{\beta} \widehat{\alpha}\right)}{\sqrt{5}}$ & $\sqrt{5} \widehat{\alpha}\left(\frac{1+\sqrt{5}}{2}\right)^{n}-\sqrt{5} \widehat{\beta}\left(\frac{1-\sqrt{5}}{2}\right)^{n}$ \\
Pell Quaternion & $\frac{(-1)^{m-1}((1-\sqrt{2}) \widehat{\alpha}-(1+\sqrt{2}) \widehat{\beta} \widehat{\alpha})}{2 \sqrt{2}}$ & $\frac{\widehat{\alpha}(1+\sqrt{2})^{n}-\widehat{\beta}(1-\sqrt{2})^{n}}{2 \sqrt{2}}$ \\
Jacobsthal Quaternion & $\frac{(-2)^{m-1}(-\widehat{\alpha} \widehat{\beta}-2 \widehat{\beta} \widehat{\alpha})}{3}$ & \\
\hline where & $\widehat{\alpha} 2^{n}-\widehat{\beta}(-1)^{n}$ \\
\hline
\end{tabular}

In the next table we give Cassini's identities and Binet formulas for some Horadam (Fibonacci, Lucas, Pell, Jacobsthal) octonions:

Table 6.

\begin{tabular}{ccc}
\hline Horadam $2^{3}$-ions: & $\widehat{W}_{m-1} \widehat{W}_{m+1}-\widehat{W}_{m}^{2}$ & Binet Formula \\
\hline Fibonacci Octonions & $\frac{(-1)^{m-1}\left(\left(\frac{1-\sqrt{5}}{2}\right) \widehat{\alpha} \widehat{\beta}-\left(\frac{1+\sqrt{5}}{2}\right) \widehat{\beta} \widehat{\alpha}\right)}{\sqrt{5}}$ & $\frac{\widehat{\alpha}\left(\frac{1+\sqrt{5}}{2}\right)^{n}-\widehat{\beta}\left(\frac{1-\sqrt{5}}{2}\right)^{n}}{\sqrt{5}}$ \\
Lucas Octonions & $\frac{-5(-1)^{m-1}\left(\left(\frac{1-\sqrt{5}}{2}\right) \widehat{\alpha} \widehat{\beta}-\left(\frac{1+\sqrt{5}}{2}\right) \widehat{\beta} \widehat{\alpha}\right)}{\sqrt{5}}$ & $\sqrt{5} \widehat{\alpha}\left(\frac{1+\sqrt{5}}{2}\right)^{n}-\sqrt{5} \widehat{\beta}\left(\frac{1-\sqrt{5}}{2}\right)^{n}$ \\
Pell Octonions & $\frac{(-1)^{m-1}((1-\sqrt{2}) \widehat{\alpha} \widehat{\beta}-(1+\sqrt{2}) \widehat{\beta} \widehat{\alpha})}{2 \sqrt{2}}$ & $\frac{\widehat{\alpha}(1+\sqrt{2})^{n}-\widehat{\beta}(1-\sqrt{2})^{n}}{2 \sqrt{2}}$ \\
$\frac{(-2)^{m-1}-\widehat{\alpha}(-1)^{n}}{3}$ & \\
Jacobsthal Octonions & $\frac{(-2 \widehat{\beta} \widehat{\alpha})}{3}$ & \\
where & $\widehat{\alpha}=\sum_{s=0}^{7} \alpha^{s} e_{s}, \quad \widehat{\beta}=\sum_{s=0}^{7} \beta^{s} e_{s}$.
\end{tabular}

In the following table we present Cassini's identities and Binet formulas for some Horadam (Fibonacci, Lucas, Pell, Jacobsthal) sedenions:

Table 7. 


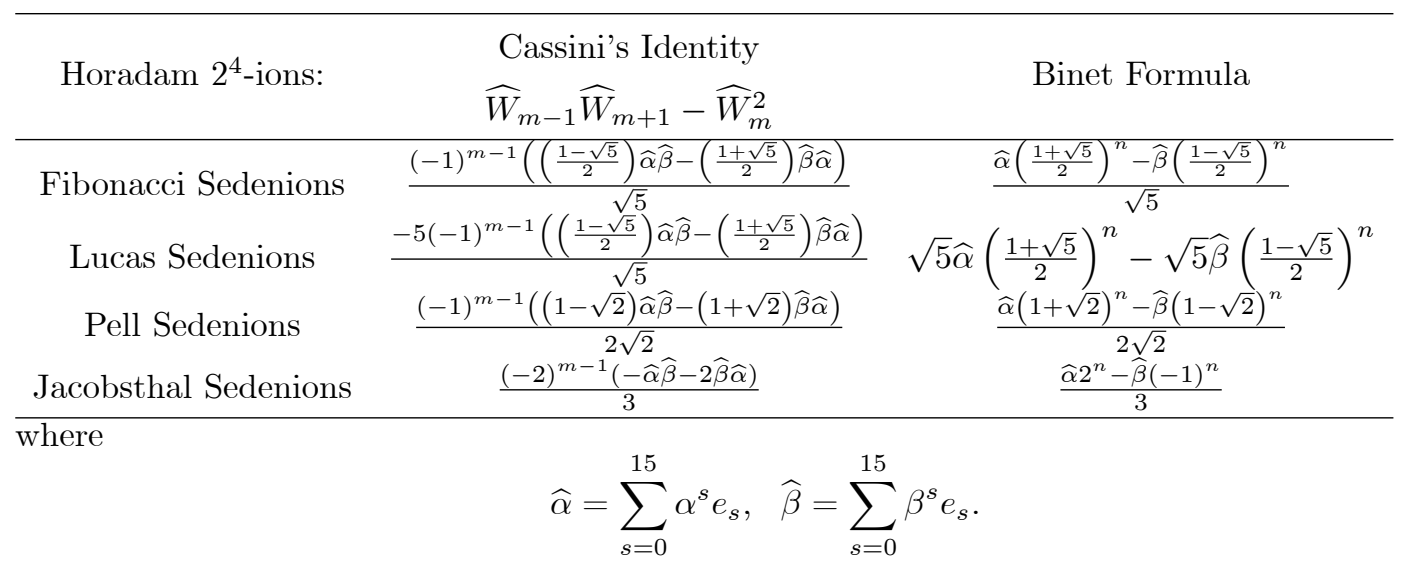

In the following table we give Cassini's identities and Binet formulas for some Horadam (Fibonacci, Lucas, Pell, Jacobsthal) trigintaduonions:

Table 8.

\begin{tabular}{ccc}
\hline Horadam $2^{5}$-ions: & \multicolumn{1}{c}{ Cassini's Identity } & Binet Formula \\
\hline Fibonacci Trigintaduonions & $\frac{\widehat{W}_{m-1} \widehat{W}_{m+1}-\widehat{W}_{m}^{2}}{2)^{m-1}\left(\left(\frac{1-\sqrt{5}}{2}\right) \widehat{\alpha} \widehat{\beta}-\left(\frac{1+\sqrt{5}}{2}\right) \widehat{\beta} \widehat{\alpha}\right)}$ & $\frac{\widehat{\alpha}\left(\frac{1+\sqrt{5}}{2}\right)^{n}-\widehat{\beta}\left(\frac{1-\sqrt{5}}{2}\right)^{n}}{\sqrt{5}}$ \\
Lucas Trigintaduonions & $\frac{-5(-1)^{m-1}\left(\left(\frac{1-\sqrt{5}}{2}\right) \widehat{\alpha} \widehat{\beta}-\left(\frac{1+\sqrt{5}}{2}\right) \widehat{\beta} \widehat{\alpha}\right)}{\sqrt{5}}$ & $\sqrt{5} \widehat{\alpha}\left(\frac{1+\sqrt{5}}{2}\right)^{n}-\sqrt{5} \widehat{\beta}\left(\frac{1-\sqrt{5}}{2}\right)^{n}$ \\
Pell Trigintaduonions & $\frac{(-1)^{m-1}((1-\sqrt{2}) \widehat{\alpha} \widehat{\beta}-(1+\sqrt{2}) \widehat{\beta} \widehat{\alpha})}{2 \sqrt{2}}$ & $\frac{(1+\sqrt{2})^{n}-\widehat{\beta}(1-\sqrt{2})^{n}}{2 \sqrt{2}}$ \\
Jacobsthal Trigintaduonions & $\frac{(-2)^{m-1}(-\widehat{\alpha} \widehat{\beta}-2 \widehat{\beta} \widehat{\alpha})}{3}$ & \\
\hline where & $\widehat{\alpha}=\sum_{s=0}^{31} \alpha^{s} e_{s}, \widehat{\beta}=\sum_{s=0}^{31} \beta^{s} e_{s}$. \\
\hline
\end{tabular}

REMARK 1. The formulas in the above last four tables looks the same but notice the differencies of $\widehat{\alpha}$ and $\widehat{\beta}$ 's for each case of $k$.

The following theorem gives d'Ocagne's identity for Horadam $2^{k}$-ions.

Theorem 5 (d'Ocagne's Identity). For any integers $m$ and $n$, we have

$$
\widehat{W}_{m} \widehat{W}_{n+1}-\widehat{W}_{m+1} \widehat{W}_{n}=\frac{A B\left(\alpha^{n} \beta^{m}-\alpha^{m} \beta^{n}\right)(\beta \widehat{\alpha} \widehat{\beta}-\alpha \widehat{\beta} \widehat{\alpha})}{(\alpha-\beta)^{2}} .
$$

Proof. By using Binet formula, we obtain

$$
\widehat{W}_{m} \widehat{W}_{n+1}-\widehat{W}_{m+1} \widehat{W}_{n}=\frac{A \widehat{\alpha} \alpha^{m}-B \widehat{\beta} \beta^{m}}{\alpha-\beta} \frac{A \widehat{\alpha} \alpha^{n+1}-B \widehat{\beta} \beta^{n+1}}{\alpha-\beta}-\frac{A \widehat{\alpha} \alpha^{m+1}-B \widehat{\beta} \beta^{m+1}}{\alpha-\beta} \frac{A \widehat{\alpha} \alpha^{n}-B \widehat{\beta} \beta^{n}}{\alpha-\beta}
$$

After necessary calculations we get the identity (3.14).

In the next Theorem, we give the summation formula for the first $n+1$ Horadam $2^{k}$-ions.

TheOREM 6. The sum formula for Horadam $2^{k}$-ions is

$$
\sum_{i=0}^{n} \widehat{W}_{i}=\frac{1}{\alpha-\beta}\left(\frac{B \widehat{\beta} \beta^{n+1}}{1-\beta}-\frac{A \widehat{\alpha} \alpha^{n+1}}{1-\alpha}\right)+C,
$$


where

$$
C=\frac{A \widehat{\alpha}(1-\beta)-B \widehat{\beta}(1-\alpha)}{(\alpha-\beta)(1-\alpha)(1-\beta)}
$$

Proof. By using the Binet formula and then geometric series we obtain

$$
\begin{aligned}
\sum_{i=0}^{n} \widehat{W}_{i} & =\frac{A \widehat{\alpha}}{\alpha-\beta} \sum_{i=0}^{n} \alpha^{n}-\frac{B \widehat{\beta}}{\alpha-\beta} \sum_{i=0}^{n} \beta^{n} \\
& =\frac{A \widehat{\alpha}}{\alpha-\beta} \frac{1-\alpha^{n+1}}{1-\alpha}-\frac{B \widehat{\beta}}{\alpha-\beta} \frac{1-\beta^{n+1}}{1-\beta}
\end{aligned}
$$

After some calculations we get

$$
\sum_{i=0}^{n} \widehat{W}_{i}=\frac{1}{\alpha-\beta}\left(\frac{B \widehat{\beta} \beta^{n+1}}{1-\beta}-\frac{A \widehat{\alpha} \alpha^{n+1}}{1-\alpha}\right)+\frac{A \widehat{\alpha}(1-\beta)-B \widehat{\beta}(1-\alpha)}{(\alpha-\beta)(1-\alpha)(1-\beta)} .
$$

This completes the proof.

By using the initial values and roots of characteristic equation we can state the norm of $n t h$ Horadam $2^{k}$-ions as follows.

THEOREM 7. The norm of nth Horadam $2^{k}$-ions is

$$
N_{r}\left(\widehat{W}_{n}\right)=\frac{A^{2} \alpha^{2 n}\left(1+\alpha^{2}+\alpha^{4}+\cdots+\alpha^{N-2}\right)+B^{2} \beta^{2 n}\left(1+\beta^{2}+\beta^{4}+\cdots+\beta^{N-2}\right)}{(\alpha-\beta)^{2}}-D
$$

where

$$
D=\frac{2 A B(-q)^{n}\left(a+(-q)+\cdots+(-q)^{\frac{N}{2}-1}\right)}{(\alpha-\beta)^{2}} \text { and } N=2^{k} .
$$

Proof. We have defined $n t h$ Horadam $2^{k}$-ions as

$$
\widehat{W}_{n}=W_{n} e_{0}+W_{n+1} e_{1}+\cdots+W_{n+N-1} e_{N-1} .
$$

Thus, norm of $n t h$ Horadam $2^{k}$-ions is

$$
N_{r}\left(\widehat{W}_{n}\right)=\widehat{W}_{n}{\widehat{W_{n}}}_{n}={\widehat{W_{n}}}_{W_{n}}=W_{n}^{2}+W_{n+1}^{2}+\cdots+W_{n+N-1}^{2} .
$$

Making necessary calculations and using the identities $\alpha+\beta=p$ and $\alpha \beta=-q$, we obtain

$$
\begin{aligned}
N_{r}\left(\widehat{W}_{n}\right)= & \frac{A^{2} \alpha^{2 n}\left(1+\alpha^{2}+\alpha^{4}+\cdots+\alpha^{N-2}\right)}{(\alpha-\beta)^{2}}+\frac{B^{2} \beta^{2 n}\left(1+\beta^{2}+\beta^{4}+\cdots+\beta^{N-2}\right)}{(\alpha-\beta)^{2}}- \\
& \frac{2 A B(-q)^{n}\left(a+(-q)+\cdots+(-q)^{\frac{N}{2}-1}\right)}{(\alpha-\beta)^{2}} .
\end{aligned}
$$




\section{Matrix Methods in Horadam $2^{k}$-ions}

We now consider the following two special cases of $\left\{W_{n}\right\}$ :

$$
\begin{aligned}
& U_{n}:=U_{n}(p, q)=W_{n}(0,1 ; p, q), \\
& V_{n}:=V_{n}(p, q)=W_{n}(2, p ; p, q),
\end{aligned}
$$

Then $\left\{U_{n}\right\}$ and $\left\{V_{n}\right\}$ can be expressed in the form

$$
\begin{aligned}
& U_{n}=\left(\alpha^{n}-\beta^{n}\right) /(\alpha-\beta), \\
& V_{n}=\alpha^{n}+\beta^{n} .
\end{aligned}
$$

These numbers $U_{n}$ and $V_{n}$ are called fundamental numbers and primordial numbers, respectively. Note that if $p=1, q=1$ then $\left(U_{n}\right)$ and $\left(V_{n}\right)$ are classical Fibonacci and Lucas sequences, i.e,

$$
\begin{aligned}
& F_{n}=W_{n}(0,1 ; 1,1)=U_{n}, \\
& L_{n}=W_{n}(2,1 ; 1,1)=V_{n} .
\end{aligned}
$$

The matrix method is very useful method in order to obtain some identities for special sequences. We define the square Horadam matrix $M$ of order 2 as:

$$
M=\left(\begin{array}{cc}
p & q \\
1 & 0
\end{array}\right)=\left(\begin{array}{cc}
U_{2} & q U_{1} \\
U_{1} & q U_{0}
\end{array}\right) .
$$

Note that

$$
M^{n}=\left(\begin{array}{cc}
U_{n+1} & q U_{n} \\
U_{n} & q U_{n-1}
\end{array}\right) .
$$

For a proof of (4.3), see [8]. We can give a Corollary of the formula (4.3).

COROllary 2. [8] For every $n \geq 1$ we have

(a): $\operatorname{det}\left(M^{n}\right)=(-q)^{n}$

(b): $U_{n+1} U_{n-1}-U_{n}^{2}=-(-q)^{n-1}$ (Cassini formula).

Writing the recurrence (2.1) as

$$
\left(\begin{array}{c}
W_{n} \\
W_{n-1}
\end{array}\right)=\left(\begin{array}{cc}
p & q \\
1 & 0
\end{array}\right)\left(\begin{array}{c}
W_{n-1} \\
W_{n-2}
\end{array}\right)
$$

in matrix form leads readily to the matrix power equation

$$
\left(\begin{array}{c}
W_{n} \\
W_{n-1}
\end{array}\right)=\left(\begin{array}{cc}
p & q \\
1 & 0
\end{array}\right)^{n-1}\left(\begin{array}{c}
W_{1} \\
W_{0}
\end{array}\right)
$$


which holds for $n \geq 1$. Also we can write

$$
\left(\begin{array}{c}
U_{n+1} \\
U_{n}
\end{array}\right)=\left(\begin{array}{ll}
p & q \\
1 & 0
\end{array}\right)\left(\begin{array}{c}
U_{n} \\
U_{n-1}
\end{array}\right)
$$

and

$$
\left(\begin{array}{c}
V_{n+1} \\
V_{n}
\end{array}\right)=\left(\begin{array}{ll}
p & q \\
1 & 0
\end{array}\right)\left(\begin{array}{c}
V_{n} \\
V_{n-1}
\end{array}\right) .
$$

Now we define the matrix $M_{W}$ as

$$
M_{W}=\left(\begin{array}{cc}
\widehat{W}_{2} & q \widehat{W}_{1} \\
\widehat{W}_{1} & q \widehat{W}_{0}
\end{array}\right) .
$$

This matrice $M_{W}$ can be called Horadam $2^{k}$-ions matrix.

THEOREM 8. For $n \geq 0$, the following holds:

$$
M_{W}\left(\begin{array}{cc}
p & q \\
1 & 0
\end{array}\right)^{n}=\left(\begin{array}{cc}
\widehat{W}_{n+2} & q \widehat{W}_{n+1} \\
\widehat{W}_{n+1} & q \widehat{W}_{n}
\end{array}\right)
$$

Proof. We prove by mathematical induction on $n$. If $n=0$ then the result is clear. Now, we assume it is true for $n=k$, that is

$$
M_{W} M^{k}=\left(\begin{array}{cc}
\widehat{W}_{k+2} & q \widehat{W}_{k+1} \\
\widehat{W}_{k+1} & q \widehat{W}_{k}
\end{array}\right) .
$$

If we use (3.2), then for $k \geq 1$, we have $\widehat{W}_{k+3}=p \widehat{W}_{k+2}+q \widehat{W}_{k+1}$ and $\widehat{W}_{k+2}=p \widehat{W}_{k+1}+q \widehat{W}_{k}$. Then by induction hypothesis, we obtain

$$
\begin{aligned}
M_{W} M^{k+1} & =\left(M_{W} M^{k}\right) M=\left(\begin{array}{cc}
\widehat{W}_{k+2} & q \widehat{W}_{k+1} \\
\widehat{W}_{k+1} & q \widehat{W}_{k}
\end{array}\right)\left(\begin{array}{ll}
p & q \\
1 & 0
\end{array}\right) \\
& =\left(\begin{array}{cc}
p \widehat{W}_{k+2}+q \widehat{W}_{k+1} & q \widehat{W}_{k+2} \\
p \widehat{W}_{k+1}+q \widehat{W}_{k} & q \widehat{W}_{k+1}
\end{array}\right)=\left(\begin{array}{cc}
\widehat{W}_{k+3} & q \widehat{W}_{k+2} \\
\widehat{W}_{k+2} & q \widehat{W}_{k+1}
\end{array}\right) \\
& =\left(\begin{array}{cc}
\widehat{W}_{(k+1)+2} & q \widehat{W}_{(k+1)+1} \\
\widehat{W}_{(k+1)+1} & q \widehat{W}_{(k+1)}
\end{array}\right)
\end{aligned}
$$

Thus, (4.6) holds for all non-negative integers $n$.

Corollary 3. For $n \geq 0$, the following holds:

$$
\widehat{W}_{n+1}=\widehat{W}_{1} U_{n+1}+q \widehat{W}_{0} U_{n}
$$

Proof. The proof can be seen by using the matricies in (4.3) and (4.5) and the identity (4.6).

Corollary 4. For $n \geq 0$, the following (Cassini formula) holds:

$$
\widehat{W}_{n+1} \widehat{W}_{n-1}-\widehat{W}_{n}^{2}=\left(\widehat{W}_{2} \widehat{W}_{0}-\widehat{W}_{1}^{2}\right)(-q)^{n-1} .
$$


Proof. From (4.6), we have

$$
\begin{aligned}
\operatorname{det}\left(\begin{array}{cc}
\widehat{W}_{n+2} & q \widehat{W}_{n+1} \\
\widehat{W}_{n+1} & q \widehat{W}_{n}
\end{array}\right) & =\operatorname{det}\left(M_{W}\right) \operatorname{det}\left(\begin{array}{cc}
p & q \\
1 & 0
\end{array}\right)^{n} \\
& \Rightarrow \widehat{W}_{n+2} q \widehat{W}_{n}-\widehat{W}_{n+1} q \widehat{W}_{n+1}=\left(q \widehat{W}_{2} \widehat{W}_{0}-q \widehat{W}_{1}^{2}\right)(-q)^{n} \\
& \Rightarrow \widehat{W}_{n+1} \widehat{W}_{n-1}-\widehat{W}_{n}^{2}=\left(\widehat{W}_{2} \widehat{W}_{0}-\widehat{W}_{1}^{2}\right)(-q)^{n-1}
\end{aligned}
$$

THEOREM 9. Let $n$ and $m$ nonnegative integers. Then we have the following relation between fundamental and primordial $2^{k}$-ions, i.e., $\widehat{U}_{n}$ and $\widehat{V}_{n}$,

$$
\widehat{V}_{n+m}=U_{m+1} \widehat{V}_{n}+q U_{m} \widehat{V}_{n-1} .
$$

Proof. If $m=0$ then

$$
\widehat{V}_{n+0}=U_{0+1} \widehat{V}_{n}+q U_{0} \widehat{V}_{n-1}
$$

which is true. Assume that the equality holds for $m \leq k$. For $m=k+1$, we have

$$
\begin{aligned}
\widehat{V}_{n+(k+1)} & =p \widehat{V}_{n+k}+q \widehat{V}_{n+(k-1)} \\
& =p\left(U_{k+1} \widehat{V}_{n}+q U_{k} \widehat{V}_{n-1}\right)+q\left(U_{(k-1)+1} \widehat{V}_{n}+q U_{(k-1)} \widehat{V}_{n-1}\right) \\
& =\left(p U_{k+1}+q U_{k}\right) \widehat{V}_{n}+q\left(p U_{k}+q U_{(k-1)}\right) \widehat{V}_{n-1} \\
& =U_{k+2} \widehat{V}_{n}+q U_{k+1} \widehat{V}_{n-1}=U_{(k+1)+1} \widehat{V}_{n}+q U_{(k+1)} \widehat{V}_{n-1} .
\end{aligned}
$$

By strong induction on $m$, this proves (4.7).

Second method. We use Theorem 1 and formula (4.1). Note that $A=b-a \beta=p-2 \beta$ and $B=$ $b-a \alpha=p-2 \alpha$. Now we have

$$
\begin{aligned}
U_{m+1} \widehat{V}_{n}+q U_{m} \widehat{V}_{n-1}= & \frac{\alpha^{m+1}-\beta^{m+1}}{\alpha-\beta} \frac{(p-2 \beta) \widehat{\alpha} \alpha^{n}-(p-2 \alpha) \widehat{\beta} \beta^{n}}{\alpha-\beta} \\
& +q \frac{\alpha^{m}-\beta^{m}}{\alpha-\beta} \frac{(p-2 \beta) \widehat{\alpha} \alpha^{n-1}-(p-2 \alpha) \widehat{\beta} \beta^{n-1}}{\alpha-\beta} \\
= & \frac{(p-2 \beta) \widehat{\alpha} \alpha^{n+m}-(p-2 \alpha) \widehat{\beta} \beta^{n+m}}{\alpha-\beta} \\
= & \widehat{V}_{n+m} .
\end{aligned}
$$

\section{References}

[1] Biss, D.K., Dugger, D., and Isaksen, D.C., Large annihilators in Cayley-Dickson algebras, Communication in Algebra, 2008.

[2] Bilgici, G., Tokeşer, Ü,. Ünal, Z., Fibonacci and Lucas Sedenions, Journal of Integer Sequences, Article 17.1.8, 20, 1-11. 2017.

[3] Cariow, A., and Cariowa, G., Algorithm for Multiplying Two octonions, Radioelectronics and Communications Systems (Allerton Press, Inc. USA), vol. 55, No 10, (2012), pp. 464-473, 2012.

[4] Cariow, A., Cariowa G., An Algorithm for Fast Multiplication of Sedenios, Information Proccessing Letters, Volume 113, Issue, 9, 324-331, 2013. 
[5] Cariow, A., and Cariowa, G., An Algoritm for multiplication of trigintaduonions, Journal of Theoretical and Applied Computer Science, Vol. 8, No. 1, pp. 50-75, 2014.

[6] Catarino, P. The Modified Pell and Modified k-Pell Quaternions and Octonions. Advances in Applied Clifford Algebras 26, 577-590, 2016.

[7] Catarino, P., k-Pell, k-Pell-Lucas and modified k-Pell sedenions, Asian-European Journal of Mathematics, 2018.

[8] Cerda, G., Matrix Methods in Horadam Sequences, Bol. Mat. 19(2), 97-106, 2012.

[9] Çimen, C., İpek, A., On Jacobsthal and Jacobsthal-Lucas Sedenios and Several Identities Involving These Numbers, Mathematica Aeterna, Vol. 7, No.4, 447-454, 2017.

[10] Çimen, C., İpek, A, On Jacobsthal and Jacobsthal-Lucas Octonions, Mediterr. J. Math., 14:37, 1-13, 2017.

[11] Gül, K., On k-Fibonacci and k-Lucas Trigintaduonions, International Journal of Contemporary Mathematical Sciences, Vol. 13, no. 1, 1 - 10, 2018.

[12] Halici, S., Karataş, A., On a Generalization for Fibonacci Quaternions. Chaos Solitons and Fractals 98, 178-182, 2017.

[13] Horadam, A.F., A Generalized Fibonacci Sequence, American Mathematical Monthly, Vol. 68, pp. 455-459, 1961.

[14] Horadam, A. F., Complex Fibonacci Numbers and Fibonacci quaternions, Amer. Math. Monthly 70, 289-291, 1963.

[15] Horadam, A. F., Basic Properties of a Certain Generalized Sequence of Numbers, The Fibonacci Quarterly 3.3, 161-176, 1965.

[16] Horadam, A. F., Special Properties of The Sequence $w_{n}(a, b ; p, q)$, The Fibonacci Quarterly, Vol. 5, No. 5, pp. 424-434, 1967.

[17] Horadam, A. F., Generating functions for powers of a certain generalized sequence of numbers. Duke Math. J 32, 437-446, 1965.

[18] Imaeda, K., Imaeda, M., Sedenions: algebra and analysis, Applied Mathematics and Computation, 115, 77-88, 2000.

[19] İpek, A., and Çimen, C., On (p,q)-Fibonacci Octonions, Mathematica Aeterna, Vol. 6, No.6, 923-932, 2016.

[20] Karataş, A., and Halici, S., Horadam Octonions. An. Şt. Univ. Ovidus Constanta, Vol. 25(3), 97-108, 2017.

[21] Keçilioglu O, Akkuş, I., The Fibonacci Octonions, Adv. Appl. Clifford Algebr. 25, 151-158, 2015.

[22] Moreno, G., The zero divisors of the Cayley-Dickson algebras over the real numbers, Bol. Soc. Mat. Mexicana (3) 4 , 13-28,1998.

[23] Polatlı, E., A Generalization of Fibonacci and Lucas Quaternions, Advances in Applied Clifford Algebras, 26 (2), 719-730, 2016.

[24] Makarov, O.M., An algorithm for the multiplication of two quaternions, U.S.S.R. Comput. MathsMath. Phys. Vol. 17, pp. 221-222, 1978.

[25] Szynal-Liana, A. and I. Wloch I., The Pell quaternions and the Pell octonions. Advances in Applied Clifford Algebras 26.1, 435-440, 2016.

[26] Tasci, D., On k-Jacobsthal and k-Jacobsthal-Lucas Quaternions, Journal of Science and Arts, year 17, No. 3(40), pp. 469-476, 2017.

Zonguldak Bulent Ecevit University,

Department of Mathematics,

Zonguldak, Turkey.

E-mail: gocenm@hotmail.com(corrresponding author), yuksel_soykan@hotmail.com 